\title{
Problemi relativi all'esposizione alla luce solare per i trapiantati di rene
}

\author{
Stefania Motta \\ Università degli Studi di Milano e Humanitas Research Hospital, Milano
}

\begin{abstract}
Kidney transplant recipients and sun exposure
Non-melanoma skin cancers induced by solar radiation are the most frequent types of cancers in humans and their incidence is increasing worldwide. Ultraviolet radiation (UV) is a major environmental cause. The individual risk of skin cancer is due both to the total cumulative exposure to UV radiation and to sporadic patterns of sun exposure as during free time.

Organ transplant recipients have a relative risk of developing squamous cell carcinoma 100 -fold higher than healthy subjects, whereas the risk of developing basal cell carcinoma is increased by 10 .

Beside the direct effect on keratinocyte DNA, UV radiations have also a negative effect on the local immunosurveillance in the exposed areas and on systemic immunity.

Educational programs on sun exposure for organ transplant recipients recommend to limit the time of exposure and to apply sunscreen creams.

Sunscreen creams are topical preparations that attenuate the effects of UV radiation on the skin. Recently the safety of sunscreen creams is a matter of discussion because of the toxicological studies performed on chemical filters.

The majority of chemical filters absorb through the skin, then into the blood. T-shirts, hats and sunglasses provide full protection from UV rays. Body areas (face, neck, ears and décolleté) exposed to solar radiation can be efficiently protected applying a blend of mineral powders (clays, bentonite, kaolin, montmorillonite) formulated to reflect sunrays. The use of mineral powders as sunscreens is encouraged also for dialysed subjects and for patients with autoimmune diseases.
\end{abstract}

Keywords: Organ Transplant recipients, Skin cancer prevention, Dyalisis, Sun Reflectant, Mineral powders, Sunscreen

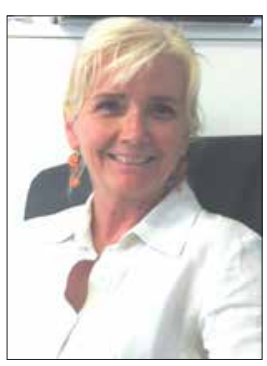

Stefania Motta

\section{Introduzione}

tumori cutanei epiteliali indotti dalla luce solare sono i tumori più frequenti nell'uomo e la loro incidenza è in aumento in ogni parte del mondo (1). La radiazione solare nella banda ultravioletta (UV) è la causa principale di questi tumori. II rischio individuale di sviluppare un tumore cutaneo epiteliomatoso è

Accepted: July 6, 2015

Published online: July 14, 2015

Indirizzo per la corrispondenza:

Stefania Motta

Università degli Studi di Milano e Humanitas

Research Hospital, Milano

stefania.motta@humanitas.it

legato sia alla dose totale cumulativa di esposizione ai raggi UV sia a quella sporadica, come quella relativa al tempo libero trascorso all'aperto (2).

I trapiantati d'organo hanno un alto tasso di sviluppo del carcinoma squamocellulare con un rischio relativo 100 volte più alto rispetto a un soggetto normale (3). In uno studio irlandese sui trapiantati renali, il $93.5 \%$ di tutti i carcinomi squamocellulari era presente nelle aree cosiddette fotoesposte (4). II carcinoma basocellulare, invece, per i trapiantati d'organo, ha un fattore di rischio 10 volte superiore rispetto al soggetto normale (5). Oltre al danno diretto sul DNA dei cheratinociti, i raggi UV hanno anche un effetto negativo sull'immunosorveglianza nelle aree fotoesposte e, probabilmente, anche sull'immunità sistemica (6). I trapiantati d'organo vanno, quindi, considerati come soggetti altamente fotosensibili al pari dei soggetti con malattie genetiche che causano il difetto di riparazione del DNA danneggiato dai raggi UV, come lo Xeroderma Pigmentoso o l'Epidermodisplasia Verruciforme. 


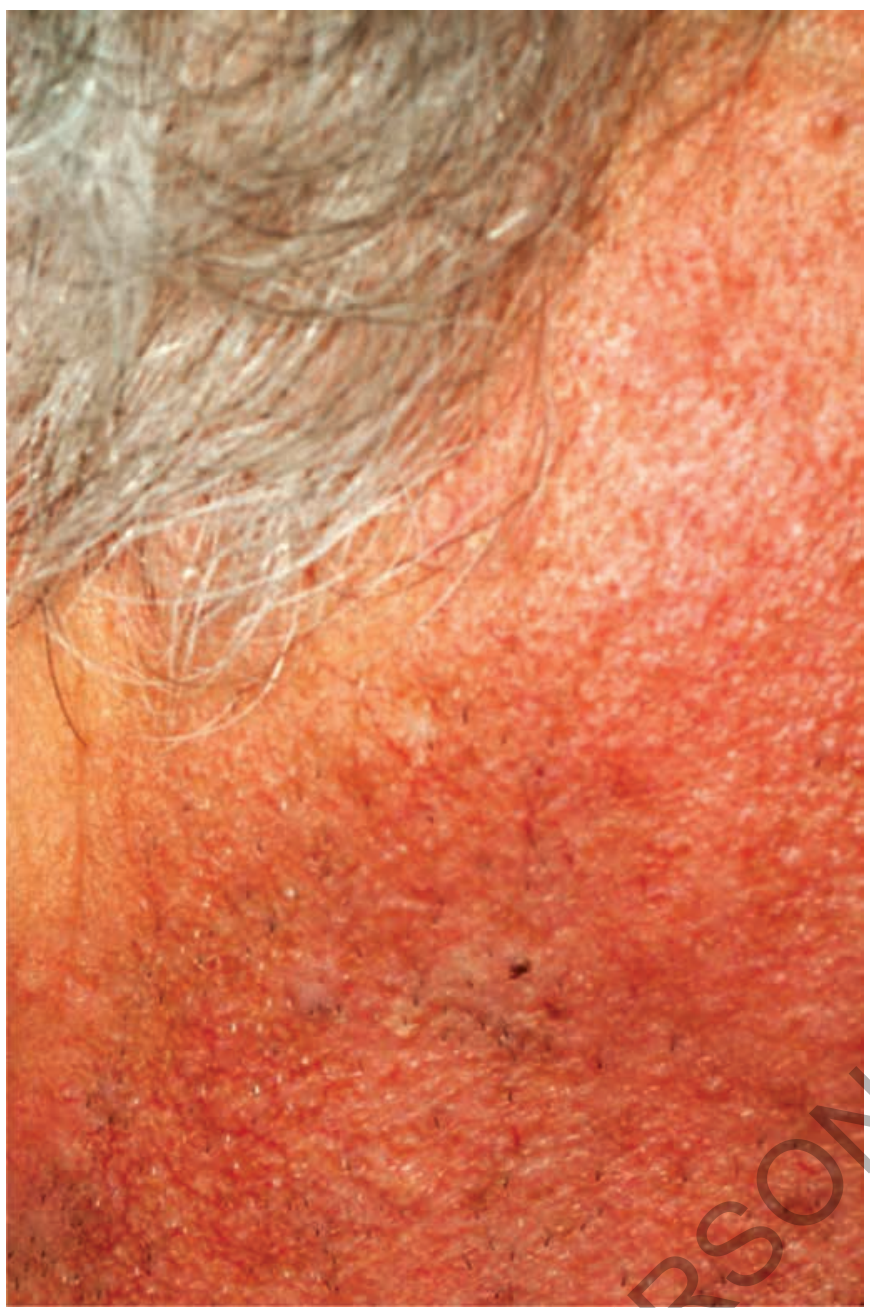

Fig. 1 - Quadro di elastosi solare.

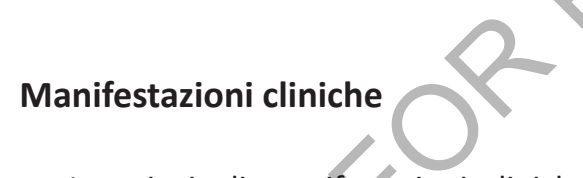

Le principali manifestazioni cliniche cutanee dovute al danno solare nel trapiantato d'organo sono:

- invecchiamento precoce

- dispigmentazione

- verrucosità diffusa

- cheratosi attiniche

- neoplasie epiteliali

L'invecchiamento precoce della cute si manifesta principalmente con il danno sulle fibre elastiche che porta al quadro dell'elastosi solare, cioè di papule giallognole, e con una vasodilatazione permanente causata dalla distruzione delle fibre elasticocollageniche perivasali (Fig. 1).

Le dispigmentazioni si manifestano sia con macchie depigmentate sia con macchie iperpigmentate e sono il segno del

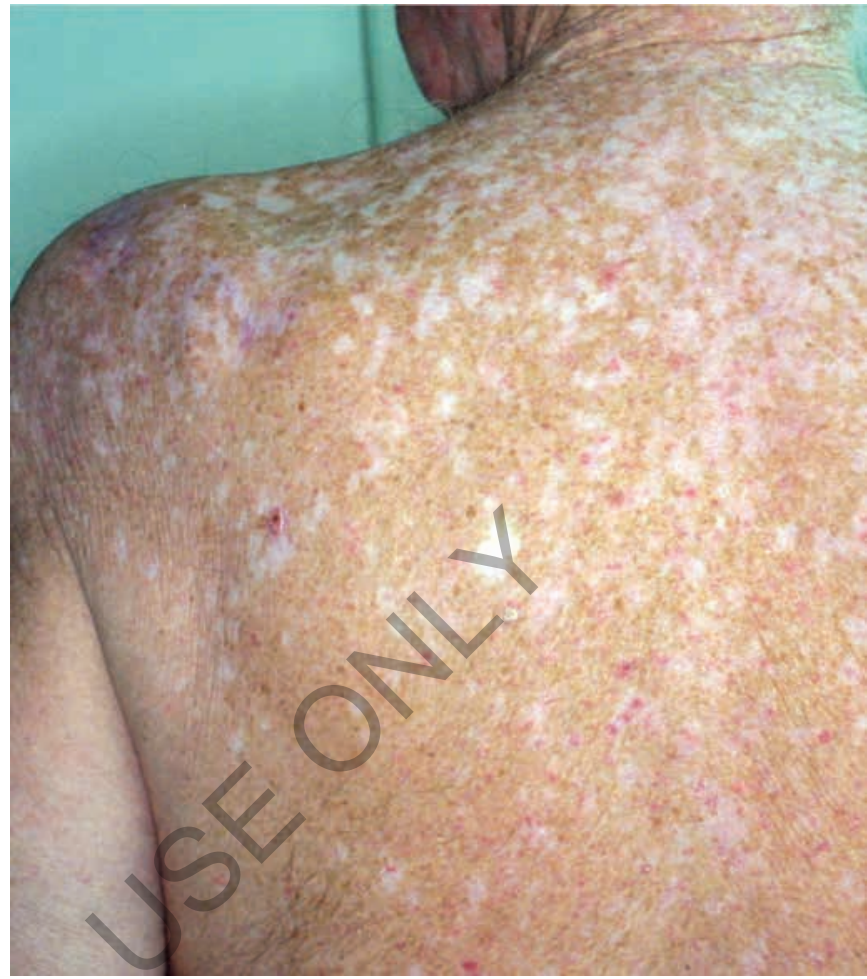

Fig. 2 -Dispigmentazioni dovute al danno solare sulla componente melanocitaria.

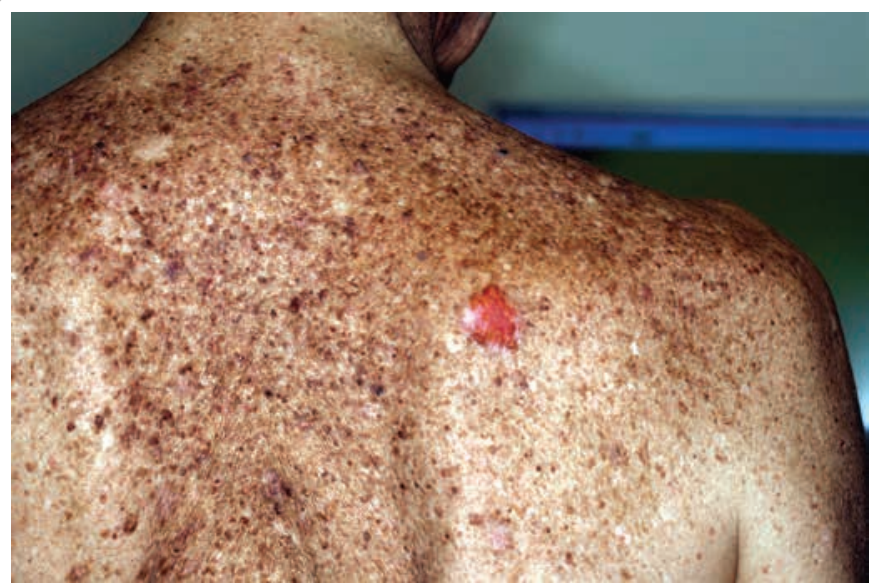

Fig. 3 - Verrucosità nelle sedi fotoesposte.

danno permanente sulla componente melanocitaria (Fig. 2).

La verrucosità diffusa si manifesta con la presenza di molteplici lesioni tipo cheratosi seborroica o cheratosi pigmentata nelle sedi più fotoesposte (Fig. 3).

Le cheratosi attiniche si manifestano come piccole aree eritematose e ipercheratosiche, di solito desquamanti (Fig. 4).

Le neoplasie epiteliali principali sono il Carcinoma Squamocellulare spesso insorto su precedente Cheratosi Attinica (Fig. 5) e il Carcinoma Basocellulare (Fig. 6). 


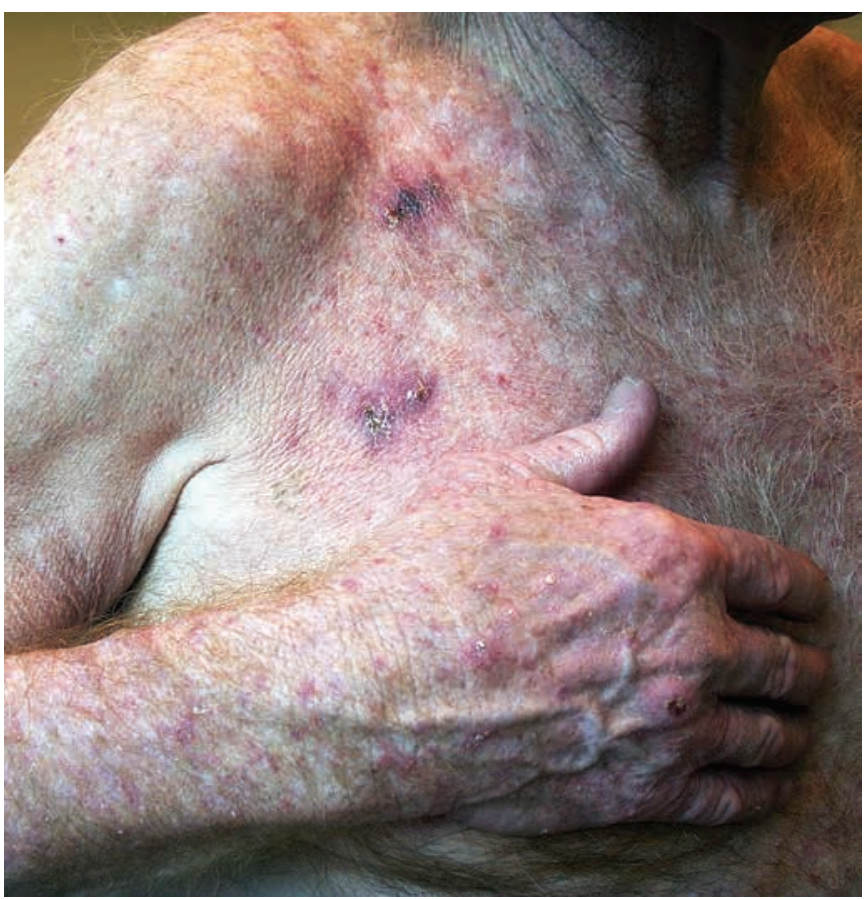

Fig. 4 - Cheratosi attiniche o solari nelle aree fotoesposte.

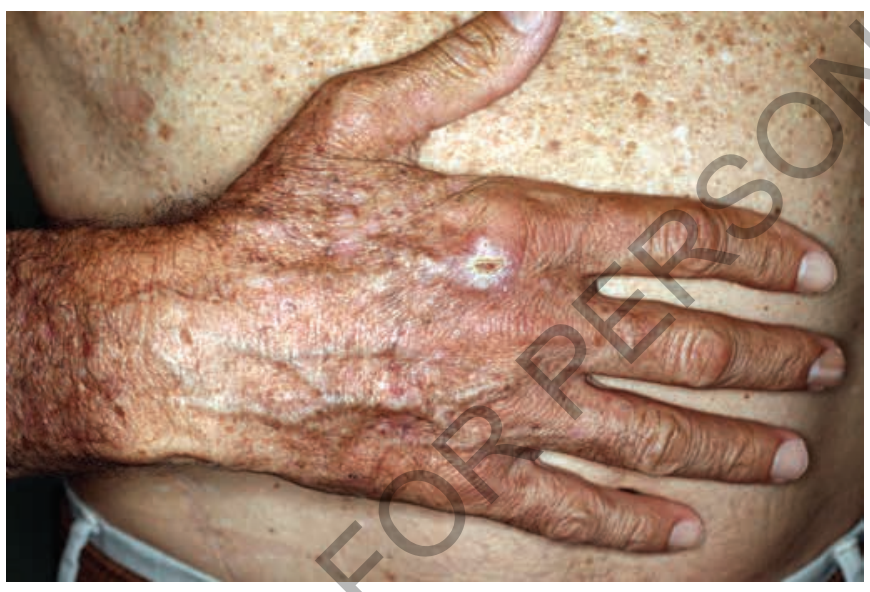

Fig. 5 - Carcinoma squamocellulare.

\section{Prevenzione}

Nei programmi educativi riguardo all'esposizione solare per i trapiantati d'organo, le principali misure preventive consigliate sono di limitare il tempo di esposizione e di applicare le creme di protezione solare (7). Queste misure, tuttavia, sono poco seguite dai trapiantati, come appare da studi specifici sul tema $(8,9)$. Per le creme solari c'è anche il dubbio se siano o meno provvedimenti validi.

Le creme solari sono preparazioni topiche in grado di attenuare gli effetti delle radiazioni UV e dovrebbero soddisfare i

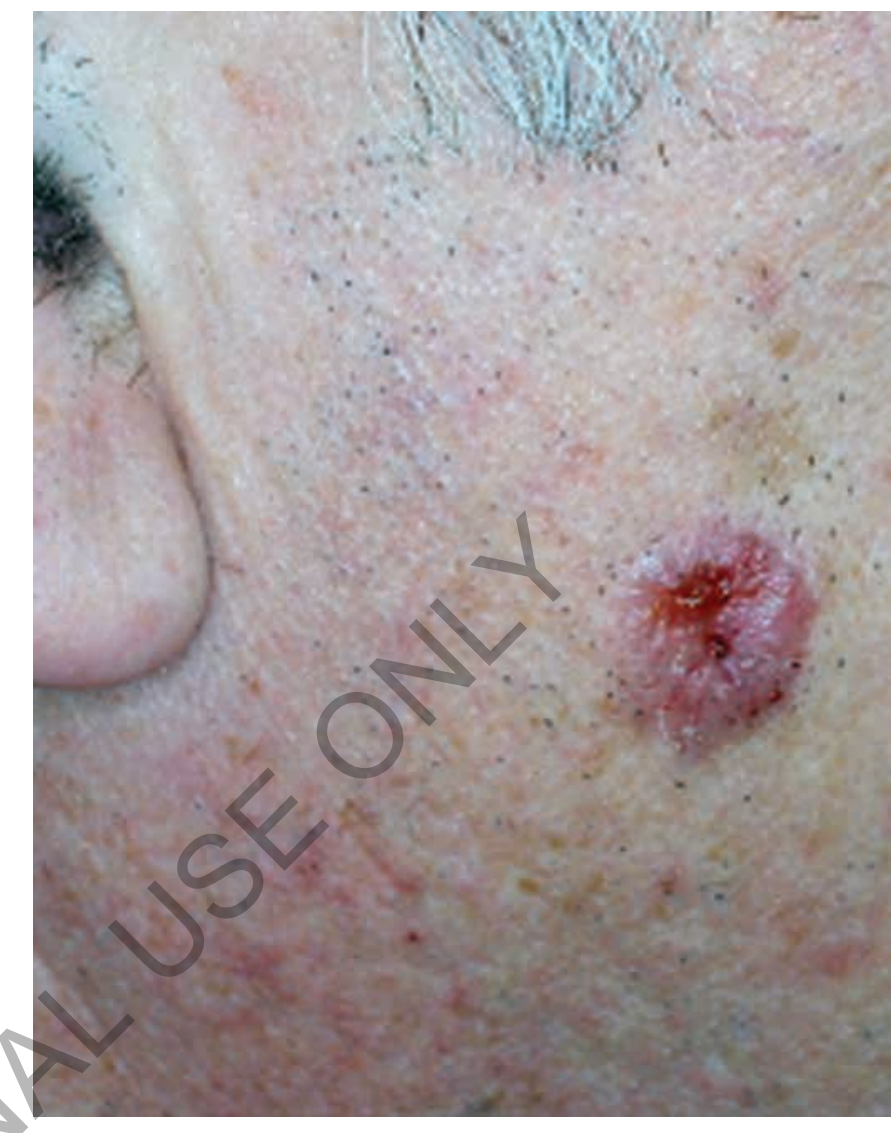

Fig. 6 - Carcinoma basocellulare.

seguenti requisiti: facili da applicare in strato uniforme, efficaci nel bloccare la maggioranza dei raggi UV, invisibili sulla pelle e non grasse e appiccicaticce. La maggior parte dei soggetti trapiantati ritiene, tuttavia, che le creme solari siano inaccettabili (10). Infatti, occorre considerare che la ciclosporina, uno degli immunosoppressori più utilizzati, induce un'iperplasia della ghiandola sebacea che porta a follicoliti seborroiche e all'acne così come anche gli steroidi. Anche altri agenti immunosoppressivi mTOR inibitori come everolimus e sirolimus sono promotori di acne; i pazienti tendono, quindi, a non applicare prodotti in crema sulla pelle, incluse le creme solari. Un altro fattore di mancata compliance è l'elevato costo delle applicazioni giornaliere delle creme solari.

Recentemente, la sicurezza delle creme solari è stata messa in dubbio dagli studi di tossicità dei filtri chimici in esse contenute. La maggioranza dei filtri chimici, a differenza di quanto si credeva, può essere assorbita dalla pelle e riversarsi nel torrente ematico. In questo passaggio, l'energia scatenata dall'eccitazione solare potrebbe danneggiare ulteriormente le cellule epidermiche; inoltre, alcuni filtri hanno un'azione estrogenica o di distruttori ormonali, mentre altri sono allergizzanti o fotoallergizzanti (11).

Un ulteriore danno è dovuto al fatto che le creme solari spingono i soggetti a esporsi per tempi più lunghi al sole, cioè 
anche dopo che l'azione filtrante si è esaurita. Per tutti questi motivi, molti gruppi e associazioni di dermatologi, a differenza delle Linee Guida, non raccomandano più l'applicazione delle creme solari. Secondo questi gruppi, la vera protezione dai danni UV consiste nel mantenere la pelle coperta con gli appositi indumenti con protezione UV, oltre che con cappello e occhiali da sole, durante tutta l'esposizione solare. Le parti che rimangono esposte alla luce, principalmente volto, orecchie, collo e décolleté e dorso delle mani e dei piedi, possono essere efficacemente protette applicando su di esse una miscela di polveri minerali ad azione fotoriflettente. Molti minerali posseggono una spiccata riflettenza solare che si esprime, oltre che nella banda UV, anche in quella del visibile.

In questa applicazione, i minerali più utilizzati sono argille, caolino, bentonite, montmorillonite, mica e ossidi di ferro.

Anche l'ossido di titanio e quello di zinco sono ottimi riflettenti solari, tuttavia, per evitare la liberazione di radicali ossigeno una volta irradiati dalla luce solare, sono stabilizzati con un rivestimento opportuno.

I fotoriflettenti minerali, quando in polvere, sono molto efficaci, mentre, quando sono dispersi in crema, perdono la maggior parte del loro effetto.

Pertanto, prima dell'esposizione solare, si raccomanda al soggetto di applicare nelle aree che rimangono esposte al sole il fotoriflettente solare in polvere. Con le moderne tecnologie di preparazione cosmetica, le polveri minerali sono fatte aderire alla pelle mediante il legame con i derivati della lisina, un composto del tutto inerte.

I vantaggi della fotoprotezione con fotoriflettente solare sono notevoli e vanno dal mancato assorbimento del prodotto da parte della pelle all'assenza di eccipienti assorbibili e di conservanti, all'inesauribilità dell'azione antisolare e alla facile rimozione attraverso il lavaggio.

\section{Conclusione}

Oltre che per i trapiantati d'organo, i dermatologi raccomandano l'applicazione dei fotoriflettenti in molte situazioni in cui le creme solari sono inopportune, come nelle allergie solari e nelle malattie autoimmuni. Anche chi è sottoposto a trattamento dialitico, anche in assenza di una pseudoporfiria, deve essere considerato soggetto maggiormente suscettibile ai danni dai raggi solari, per cui valgono le raccomandazioni di fotoprotezione consigliate per i trapiantati d'organo. Per i motivi elencati, il gruppo di dermatologia dell'Humanitas Research Hospital ha abolito la prescrizione di creme solari sostituendola con quella dei fotoriflettenti minerali.

L'Autore auspica che la modalità di fotoesposizione e protezione con indumenti e l'applicazione di fotoriflettenti minerali siano adottate nelle prossime Linee Guida per i soggetti trapiantati d'organo.

\section{Disclosures}

Financial support: No financial support was received for this submission.

Conflict of interest: The author has no conflict of interest.

\section{Bibliografia}

1. Boyle P, Doré JF, Autier P, Ringborg U. Cancer of the skin: a forgotten problem in Europe. Ann Oncol. 2004;15(1):5-6.

2. Ackerman AB, Mones JM. Solar (actinic) keratosis is squamous cell carcinoma. Br J Dermatol. 2006;155(1):9-22.

3. Lindelöf B, Sigurgeirsson B, Gäbel H, Stern RS. Incidence of skin cancer in 5356 patients following organ transplantation. $\mathrm{Br} J$ Dermatol. 2000;143(3):513-9.

4. Moloney FJ, Almarzouqi E, O'Kelly P, Conlon P, Murphy GM. Sunscreen use before and after transplantation and assessment of risk factors associated with skin cancer development in renal transplant recipients. Exp Dermatol. 2002;11(Suppl. 1): 9-12.

5. Kricker A, Armstrong BK, English DR, Heenan PJ. Does intermittent sun exposure cause basal cell carcinoma? a case-control study in Western Australia. Int J Cancer. 1995;60(4):489-94.

6. Schwarz A, Schwarz T. Molecular determinants of UV-induced immunosuppression. Arch Dermatol. 2005;141(8):978-82.

7. Clowers-Webb HE, Christenson LJ, Phillips PK, et al. Educational outcomes regarding skin cancer in organ transplant recipients: Randomized intervention of intensive vs standard education. Arch Dermatol. 2006;142(6):712-8.

8. Kasiske BL, Vazquez MA, Harmon WE, et al. Recommendations for the outpatient surveillance of renal transplant recipients. J Am Soc Nephrol. 2000;11(Suppl. 15):S1-86.

9. Robinson JK, Rigel DS. Sun protection attitudes and behaviors of solid-organ transplant recipients. Dermatol Surg. 2004;30 (4 Pt. 2):610-5.

10. Ulrich C, Degen A, Patel MJ, Stockfleth E. Sunscreens in organ transplant patients. Nephrol Dial Transplant. 2008;23(6):1805-8.

11. http://draxe.com/75-of-sunscreens-are-toxic-what-to-do-instead/. 